stomach: We bathe the babies at least twice a day, but very, very gently, moving them as little as possible. The bath often seems to allay the thirst to some extent and always the restlessness. Except for the bath we keep the baby in absolute seclusion. We choose the quietest corner of the ward and we only disturb him when absolutely necessary. The nurse attends to him before feeding and immediately the feeding is completed the curtain is drawn over the child-we keep all bad diarrhoea and vomiting cases in curtained beds-and the baby is left "in retreat" until the next feed, if possible. The mouth of these patients requires a great deal of attention, and nothing keeps it better than ordinary cold water, or slightly alkaline water (sodium bicarbonate one grain to the ounce). It must always be washed out before food and-if we can risk it without exciting fresh vomiting-also afterwards. Medicated mouth washes are generally harmful through having too decided a taste, so causing a recurrence of sickness. The period when the care of the mouth is most important is while intracellular feeding only is being carried on. The swabbing of the mouth then does not only serve the purpose of keeping the part clean but also of allaying the thirst and the accompanying restlessness. We must not, however, allow ourselves to give in to the baby's evident craving for fluid which he shows by eagerly sucking the wet rag we are using and let him have a drink of water, because this misplaced pity has again and again re-started the barely arrested vomiting. Another point which the nurse must be extremely careful about is the thorough scrubbing and disinfecting of her own hands after having attended to the infant. This is most essential, as by infecting the baby's food with even the smallest particle of fæcal matter the sickness, which has just been stopped with great difficulty, may be started again. So much importance is attached to this matter in some children's hospitals on the continent that special nurses are told off to do the feeding, while others attend to the cleaning of the patients.

The above régime applies exclusively to acute cases. For chronic cases of diarrhoe and vomiting who cannot retain milk and milk mixtures, we have found malt soup very satisfactory. Babies can be kept on this food for as long as three weeks and then gradually be trained to take ordinary milk, generally viâ Benger's food. Horlick's malted milk also has acted fairly well in some cases of this kind. A new preparation, "pegnin," which has the great advantage of reducing the casein in cow's milk to a very fine, flocculent curd, promises to become a valuable addition to the infant's dietary. Another experience which we have had in obstinate cases of habitual vomiting is that some will not reject their food if it is introduced by the nasal tube, whereas all attempts at feeding by the mouth are useless. If to the treatment of chronic cases we could add sunshine and good, fresh, bracing country air, we might hope to carry it out with very much more chance of success. I append the formulæ of the foods above mentioned hoping they may prove useful.

Albumin mixture.-Beat up the white of two fresh eggs and add gradually one pint of cold, sterile normal saline solution. Stir up well and strain through muslin. Keep cold.

Beef-juice (Guy's Hospital Pharmacopœia).-One pound of lean beef is finely chopped and added to one pint of water. This mixture is then gently heated (being constantly stirred) until the temperature is $55^{\circ} \mathrm{C}$. $\left(130^{\circ} \mathrm{F}\right.$.) at which it is kept for 15 minutes. To this are added three ounces of beef-tea and a small teaspoonful of salt. The whole is then strained through muslin over a hair sieve and filtered into a flask in which it is to be kept. If placed in an ice-box the juice will keep for two or three days.

Raw meat-juice.-One pound of lean beef is finely chopped. Add to it five ounces of water, mix up well, and stand on ice for three hours. Then strain through muslin, the meat being well squeezed. Keep in the ice-box.

Malt soup. -50 grammes (13 drachms) of wheat flour are well mixed up with one-third litre (12 ounces) of fresh cow's milk and the mixture is passed through a hair sieve. In another vessel 100 grammes (three ounces two drachms) of extract of malt are dissolved in two-thirds of a litre (24 ounces) of water at $50^{\circ} \mathrm{O} .\left(122^{\circ} \mathrm{F}\right.$.) and to this are added ten cubic centimetres (two and a half drachms) of an 11 per cent. solution of potassium carbonate. The two preparations are then mixed and boiled up quickly.

Knorr's Hafermehl.-Supplied by Messrs. Allman, 9, Cullum.street, Fenchurch-street.

Pegnin.-Supplied by Farbwerke, 51, St. Mary-axe, E.C.

\section{SUCCESSFUL TREATMENT OF SPORADIC DYSENTERY BY APLOPAPPUS BAYLAHUEN.}

\author{
BY WILLIAM FINGLAND, L.R.C.P. \& S. EDIN.
}

IT falls to the lot of most practitioners to have from time to time to treat cases of sporadic dysentery, colitis, or one of its protean forms, and when a more or less chronic character is assumed no disease is so rebellious to treatment. And when we consider the grave pathological condition of the colon this is not a matter of much surprise. Briefly, the mucous membrane is thickened, œdematous, congested, and cyanotic; the eminences and depressions on its surface are practically obliterated. It is bathed in a muco-serous exudation and its surface is covered with hæmorrhagic petechiæ and suppurating foci. For the amelioration and cure of these conditions many remedies have been suggested, which I will mention later, but from collected experience the disease tends to pursue a chronic and incurable course.

The drug which I wish to introduce to the notice of the profession is indigenous to South America and in Chili and other provinces of the west coast of South America it is considered a specific for this disease. It is called locally hysteronica and its botanical name is Aplopappus Baylahuen. Messrs. Parke, Davis, and Co, of London have prepared and supplied me with a fluid extract thereof. The plant belongs to the class of balsams. The fluid extract is a dark green gummy fluid, of a syrupy consistence, and has a peculiar odour. It is not miscible with water and requires to be prescribed alone and not in combination. The dose is 20 minims thrice daily, suspended in a tablespoonful of cream, milk, or freshly prepared almond emulsion. During the exhibition of this drug the diet must be carefully supervised, all vegetables and fruits prohibited, and a diet of beef, mutton, fish, and light farinaceous dishes enjoined, with hot water and milk to drink, the latter with some caution. In the three cases (each possessing in a marked degree the typical symptoms of this disease) which have come under my notice and presently to be described the curative effects of this drug were so conspicuous that I hope other members of the profession, with more frequent opportunities, will give this drug a more extended trial, as judging from $\mathrm{my}$ own success it would appear to be a specific for this troublesome malady.

CASE 1. - The patient was a lady who was believed to have contracted the disease from drinking water when staying at Heswall, Cheshire (before the present watersupply was introduced). She has had many attacks during the last ten years, each attack increasing in severity. The symptoms were quite usual-nausea, vomiting, thirst, pain, tenesmus, tormina, with incessant diarrhca accompanied with scanty muco-purulent dejecta and copious hæmorrhages. The last attack occurred two and a half years ago, when all the usual remedies were prescribed, including ipecacuanha, magnesium sulphate, and the newer astringents such as tannoform, tannigen, and fortoin. The colon was also irrigated with silver nitrate (which had formerly afforded good results), starch and opium, suprarenal extract in thin gruel, and other soothing astringents and sedatives with very little benefit. Hysteronica was prescribed for the first time, when all the symptoms gradually disappeared and the patient has now been free from any recurrence for two and a half years.

CASE 2. - A nurse in a Liverpool hospital contracted the disease when on a visit to Scarborough. She suffered from similar symptoms to the preceding, with very brief intermissions for four years. Latterly the anæmia and asthenia were pronounced, she was practically bedridden, and contemplated giving up her occupation. She had been under the care of several members of the staff of that institution but did not show any progress towards recovery. I mentioned this drug to her then medical attendant and induced him to presc ibe it. After taking a few doses the improvement was marked and continuous, her usual health and vigour being rapidly restored. About 18 months have now elapsed without any return of the symptoms.

CASE 3.-An engineer aboard a steamship trading ketween Liverpool and the West Indies stated that he had contracted the disease from using impure drinking water in those 
islands. His symptoms were similar to the above but not so severe as entirely to prevent him from following his work. I had tried magnesium sulphate but with no benefit, when I prescribed hysteronica which rapidly removed all symptoms of the disease. I last saw this patient on June 29th, 1902, when he was quite free from any recurrence and $I$ have since been informed that he has remained in perfect health.

Liverpool.

\section{A CASE OF TRAUMATIC TETANUS TREATED WITH ANTITOXIN; DEATH.}

BY H. S. ELWORTHY, F.R.C.S. ENG.,

CASUALTY OFFICER TO THE GREAT NORTHERN CENTRAL HOSPITAT.

TeTANUS, fortunately not a common disease in this country, is one of those which still maintain a very high mortality and therefore any therapeutic measures that may be taken to cure it must be of great interest to the medical profession, whether successful or otherwise. In the days before antitoxin the mortality from traumatic tetanus was something like 80 or 90 per cent., and at the present time, so far as I can gather, it amounts to about 50 per cent. or more, as unfavourable cases are probably not so often recorded as successful ones.

The case I have to relate was that of a boy, aged 14 years. He had had more than the usual share of illnesses and accidents and was not robust. He "picked at his food" and was difficult to feed at all times, was slight of build, and what one would describe as a little delicate. While playing in a cricket field on June 27 th he stepped on a spike of some kind which perforated the sole of the left foot. Medical aid was not sought for. The wound suppurated and apparently got well, so that no more was thought about it. On July 2nd he fell down on his back but made no complaint of it. On the 4th he began to complain of pains in the back, but thinking it was only a little rheumatic stiffness he went away and spent the day with a relative. On the morning of the 5th, however, he found that he could not open the mouth properly and had spasms of the muscles that caused him to bend forwards and several times he fell down on his hands and knees.

When I saw him for the first time he was walking about the room and a spasm occurred about every five minutes, causing the head and back to bend forwards. The pain was not severe and passed off in a few seconds. He declined to lie down as that caused more pain. He bit his tongue slightly in one of the spasms. The mouth opened about half an inch. There was no tenderness and the pains were about the trapezii muscles, none about the jaws. On examining the foot a small piece of grit, like cinder, and of about the size of a millet seed, was found imbedded in the skin lying in a smooth cavity. This was removed and the wound was dressed with a 1 in 20 solution of carbolic acid. The parents had given him a dose of liquorice powder without result. Three grains of calomel were given at once and a mixture of bromide and chloral was ordered to be taken every three hours. At 3 P.M. the spasms were still recurring about every five minutes. He had tried to sleep but was awakened in about ten minutes by a spasm; or else just before becoming unconscious he got clonic contractions of the hands and arms. He was becoming tired. The abdominal muscles were rigid, as were also those of the back. The spasms caused the expulsion of large quantities of flatus. By 6 P.M. I had procured through a druggist some tetanus antitoxin from the Jenner (now Lister) Institute of Preventive Medicine. As it was on Sunday the special messenger found some difficulty in getting it, having to try at sereral places before he could obtain any, and this caused some delay. Mr. E. H. Hare, who saw the patient in consultation and rendered valuable assistance on several occasions during the illness, administered chloroform while I excised the wound from the sole, swabbed the base with chloride of zinc, 40 grains to the ounce, and injected ten cubic centimetres of the antitoxin under the skin of the abdomen. He slept for about one hour after this and then the spasms returned as before. I could see no improvement, although the patient said that the pains were less severe but more frequent. His face was red, the skin was sweating, the pulse was irregular (just after a spasm) but of fair volume, and the temperature was $98.8^{\circ} \mathrm{F}$. At midnight the spasms were becoming more frequent, so another injection of ten cubic centimetres of antitoxin was made under the skin. He slept better-as much as 20 minutes at a time between the spasms-and felt better. Urine was passed into the bed on several occasions, so there was no occasion to use a catheter.

On the 6th at 10.30 A.M. a third injection was made into the left thigh and the foot was dressed as the bandage had become loose. At 12.40 P. M. he seemed quieter but attempts to feed him by the mouth brought on the spasms. The head was now retracted and the spasms caused arching backwards but were never severe nor was there any risus sardonicus at any time. At 5 P.M. he had been asleep for an hour after taking the medicine. He was then taking 15 grains of bromide, 15 grains of chloral, and five minims of tincture of cannabis indica every three hours, but the spasms now returned just as frequently, sometimes at intervals of five or six seconds. He was again put under chloroform and ten cubic centimetres of antitoxin from another source were injected into the spinal canal between the third and fourth lumbar vertebræ and the foot of the bed was raised nine inches so that it should gravitate towards the medulla. Half an hour afterwards he had a succession of spasms coming on every few seconds. He then slept for an hour, having occasional spasms at intervals of from 20 to 40 minutes. He could now turn on his side, which he could not do before owing to rigidity of the arms. He paid no attention to sounds, so that a smoking concert across the road had no effect on him nor the accidental slamming of the door by the wind. Ten grains of calomel were administered as the bowels had not acted.

On the 7th, at 9 A.M., the patient had had a fairly good night. The bowels had not been opened. The spasms came on severely from 8 to $8.30 \mathrm{~A}$. M. ; they were more sudden and jerky and caused a sort of cry from the contraction of the abdominal muscles expelling air from the lungs. There were clonic spasms as well. Food could not be taken by the mouth. He was again chloroformed and a second injection was made into the spinal canal at $9.45 \mathrm{~A}$.M. A stomach tube was passed and an egg with five ounces of milk and some meat extract were given and a nutrient suppository was inserted into the rectum. The foot of the bed was raised as before. Some antitoxin was also applied locally to the foot. The spasms beg in again at 11 A.M. and became rapid and more severe at $11.15 \mathrm{~A} . \mathrm{M}$. ; there was a succession of about 16 in a short interval. He was sweating, the pulse was 96 and fairly regular, the respiration was 46 and rather shallow, and the temperature was $101 \cdot 2^{\circ}$. At 12.50 P.M. medicine was passed through a funnel and tube into the mouth. He could swallow with difficulty. On one occasion the right eye was found deviating outwards. At 1.25 P. M. no improvement was noticed and a sixth injection of antitoxin was given under the skin. From the morning until the end chloroform was given at each spasm, sufficient to relax the muscles. They were occurring from every 10 to 15 minutes. In the afternoon the features looked drawn and pinched. The patient was fed through the tube every five hours with milk, egg, and extract of meat, and when he could not swallow the medicine it was given him with the food. At 5.30 P.M. he began to have the appearance of septic fever. As the bowels had not acted since the commencement of the illness a turpentine enema was given and was returned only a little stained by fæces. At 7.30 P. M. he collapsed and appeared to be moribund; the pulse was intermittent, irregular, and feeble, and the breathing was jerky and shallow. The eyes were semiclosed and the lips and the tongue were dry. The patient however, rallied and at 8.30 P.M. was given a nutrient enema which was expelled about ten minutes afterwards, just as the suppositories were. He also involuntarily expelled his evacuations with some force after this. The spasms occurred now at intervals of from five to eight minutes. The temperature was $104^{\circ}$ and he was sponged at intervals, which brought it down to $102.8^{\circ}$ by $11.20 \mathrm{P} \mathrm{M}$. The skin over the bony parts was red and threatening bed sores. It was rubbed with spirit each time he was turned over.

On the 8th he was fed again at 1.40 A $\mathrm{Mr}$. and some opium, digitalis, and strophanthus were added to the chloral mixture. On passing the stomach tube it was firmly gripped by muscular contraction. The spasms cortinued with some 